\title{
A Waste-derived Lithium Metasilicate Basic Catalyst
}

\author{
Victoria Katherine Elmes ${ }^{\mathrm{a}}$, Andrew Paul Mendham and Nichola Jayne Coleman \\ Faculty of Engineering and Science, University of Greenwich, Chatham Maritime, Kent, ME4 4TB, UK
}

\begin{abstract}
A mixed product of lithium metasilicate (74\%) and minor proportions of calcium carbonate, lithium carbonate and calcium hydroxide was prepared by hydrothermal synthesis from waste green container glass in $4 \mathrm{M}$ lithium hydroxide solution at $125^{\circ} \mathrm{C}$. The reaction product was characterised by powder X-ray diffraction and Fourier transform infrared spectroscopy. The lithium metasilicate product was found to be a successful heterogeneous catalyst for the Knoevenagel synthesis of ethyl trans- $\alpha$-cyanocinnamate from benzaldehyde and ethyl cyanoacetate.
\end{abstract}

\section{Introduction}

Soda-lime-silica glass (SLSG) containers form a large proportion of the urban waste-stream in every developed nation. A number of projects have been carried out to 'upcycle' surplus soda-lime-silica glass into potential value-added materials such as ion-exchangers, lightweight aggregates and ceramics [1-4]. In this respect, a recent study reports the hydrothermal synthesis of lithium metasilicate $\left(\mathrm{Li}_{2} \mathrm{SiO}_{3}\right)$ from waste container glass and its potential to act as a carrier for zinc ions [2]. Lithium metasilicate is a synthetic alkaline inosilicate with applications as a breeder material for tritium, ionic conductor, gas-sensor and $\mathrm{CO}_{2}$-sorbent $[5,6]$.

Basic inorganic waste-derived heterogeneous catalysts are currently being considered for various organic reactions [7]. These include a wide range of mixed oxide materials from fly ashes, slags and red muds, silica-rich phases from slags and rice husk ash, and calcium-rich phases derived from bones, molluscs and egg shells [7]. In particular, lithium metasilicate, prepared by calcination of rice husk ash and lithium carbonate, has proven successful in the catalysis of the transesterification of soybean oil with methanol to produce biodiesel [8].

The Knoevenagel condensation reaction of an aldehyde or ketone with an activated methylene group is widely employed in the chemical and pharmaceutical industries for the formation of carboncarbon bonds [9]. This reaction is generally carried out in solution under basic homogeneous catalysis. Environmentally benign solid basic catalysts have been proposed as a superior alternative to the traditional homogeneous catalysis for the Knoevenagel reaction which presents problems associated with the separation of the product and the disposal of the large volume of caustic waste liquor [9].

In this study, a lithium metasilicate-bearing mixed oxide product was prepared hydrothermally from waste SLSG in $4 \mathrm{M}$ lithium hydroxide solution at $125^{\circ} \mathrm{C}$ and characterised by powder X-ray diffraction analysis (XRD) and Fourier transform infrared spectroscopy (FTIR). The possibility of

\footnotetext{
${ }^{\mathrm{a}}$ Corresponding author: Vickie.elmes@gmail.com
} 
using this lithium metasilicate product as a basic heterogeneous catalyst for the Knoevenagel reaction between benzaldehyde and ethyl cyanoacetate was explored.

\section{Materials and methods}

\subsection{Preparation and characterisation}

Impure lithium metasilicate was prepared from discarded green soda-lime-silica glass (SLSG) bottles by adapting the hydrothermal method described in reference 2 . In brief, $3.0 \mathrm{~g}$ of SLSG and $60 \mathrm{~cm}^{3}$ of $4 \mathrm{M} \mathrm{LiOH}_{(\mathrm{aq})}$ were heated at $125^{\circ} \mathrm{C}$ in hermetically sealed PTFE reaction vessels under autologous pressure for 14 days. The oxide analysis of the glass was determined by X-ray fluorescence spectroscopy $[1,2]$ and is given in Table 1 . The reaction was carried out in triplicate and the products were washed with deionised water to $\mathrm{pH} \sim 10$ and dried to constant mass in air at $40{ }^{\circ} \mathrm{C}$ prior to analysis and use.

Table 1. Oxide analysis of waste green glass.

\begin{tabular}{|c|c|}
\hline Oxide component & Mass \% \\
\hline $\mathrm{SiO}_{2}$ & 72.15 \\
\hline $\mathrm{Na}_{2} \mathrm{O}$ & 13.21 \\
\hline $\mathrm{CaO}$ & 10.48 \\
\hline $\mathrm{Al}_{2} \mathrm{O}_{3}$ & 1.48 \\
\hline $\mathrm{MgO}$ & 0.94 \\
\hline $\mathrm{K}_{2} \mathrm{O}$ & 0.59 \\
\hline $\mathrm{Fe}_{2} \mathrm{O}_{3}$ & 0.46 \\
\hline $\mathrm{SO}_{3}$ & 0.28 \\
\hline $\mathrm{Cr}_{2} \mathrm{O}_{3}$ & 0.27 \\
\hline
\end{tabular}

The reaction products were analysed by powder XRD using a Bruker D8 diffractometer with $\mathrm{Cu}$ $\mathrm{K} \alpha=1.5406 \AA$, a step size of $0.02^{\circ}$ in the $2 \theta$ range from 5 to $60^{\circ}$ and a measuring time of $1 \mathrm{~s}$ per step. $\mathrm{X}$-ray diffraction data were compared with JCPDS files using DIFFRAC.EVA software (Bruker, Germany). Quantitative analysis was carried out by Rietveld refinement using a fundamental parameters approach with full axial model using TOPAS 4.2 software (Bruker, Germany) [10]. The lithium metasilicate structure used for the quantitative analysis was described by Tang et al. [11] and all other structures were selected from the TOPAS 4.2 structure database (Bruker, Germany). The FTIR spectrum of the reaction products was acquired using a Perkin Elmer Spectrum Two spectrometer between 450 and $4000 \mathrm{~cm}^{-1}$ wavenumbers, with 16 scans at a resolution of $4 \mathrm{~cm}^{-1}$.

\subsection{The Knoevenagel condensation reaction}

The catalytic properties of lithium metasilicate were investigated using the Knoevenagel condensation reaction between benzaldehyde and ethyl cyanoacetate to produce ethyl trans- $\alpha$-cyanocinnamate [9, 12]. $0.25 \mathrm{~g}$ of lithium metasilicate were added to a mixture of benzaldehyde $(19.6 \mathrm{mmol})$ and ethyl cyanoacetate $(18.8 \mathrm{mmol})$ in $50 \mathrm{~cm}^{3}$ of ethanol, in a three-necked round bottom flask, equipped with a reflux condenser, thermometer and magnetic stirrer. The mixture was heated at $80{ }^{\circ} \mathrm{C}$ for $1.5 \mathrm{~h}$, under constant stirring. At time intervals of $0,15,60$ and $105 \mathrm{mins}, 1 \mathrm{~cm}^{3}$ aliquots of the reaction mixture were withdrawn for analysis via gas chromatography (GC), using an Agilent 7683B instrument equipped with an HP-1 capillary column and a flame ionisation detector (FID). The same reaction was conducted in the absence of the lithium metasilicate product. All reactions were carried out in 
triplicate. The ethyl trans- $\alpha$-cyanocinnamate product was also confirmed by gas chromatography with mass spectrometry (Perkin Elmer turbomass DB5 spectrometer).

\section{Results and discussion}

\subsection{Characterisation}

The powder XRD pattern of the hydrothermal reaction product of waste green container glass and $4 \mathrm{M}$ aqueous lithium hydroxide solution at $125{ }^{\circ} \mathrm{C}$ for 14 days is shown in Fig. 1. These data confirm that the principal reaction product is orthorhombic lithium metasilicate (JCPDS file 29-0828), and that calcite, lithium carbonate and calcium hydroxide, are also present in minor proportions. The relative proportions of the components of the lithium metasilicate product are listed in Table 2 . The weighted profile R-factor (Rwp), for the refinement was 8.15, which demonstrates that the fittings are satisfactory.

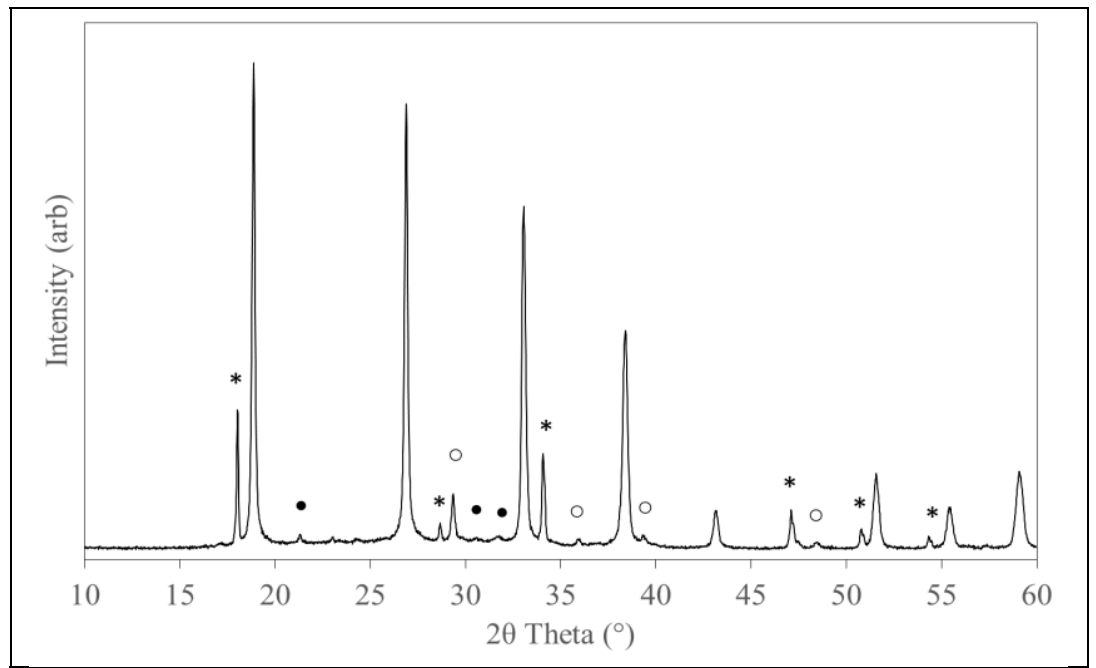

Figure 1. XRD pattern for the hydrothermal reaction product $\left(\mathrm{Key}: \bullet \mathrm{Li}_{2} \mathrm{CO}_{3} ; \circ \mathrm{CaCO}_{3} ;{ }^{*} \mathrm{Ca}(\mathrm{OH})_{2}\right)$.

Table 2. Phase composition of lithium metasilicate product.

\begin{tabular}{|c|c|}
\hline Phase & Mass (\%) \\
\hline $\mathrm{Li}_{2} \mathrm{SiO}_{3}$ & 73.52 \\
\hline Amorphous phase & 17.05 \\
\hline $\mathrm{CaCO}_{3}$ & 2.35 \\
\hline $\mathrm{Li}_{2} \mathrm{CO}_{3}$ & 2.74 \\
\hline $\mathrm{Ca}(\mathrm{OH})_{2}$ & 4.34 \\
\hline
\end{tabular}

The composition of the impure lithium metasilicate obtained in this study is similar to that reported previously for a hydrothermal reaction product of colourless SLSG synthesised in $4 \mathrm{M}$ lithium hydroxide at $100{ }^{\circ} \mathrm{C}$ for 5 days [2]. The increase in reaction temperature and time to $125{ }^{\circ} \mathrm{C}$ and 14 days, respectively, is seen to increase the proportion of lithium metasilicate from 67.9 to $73.5 \%$, and to eliminate the minor reaction product, tobermorite. 


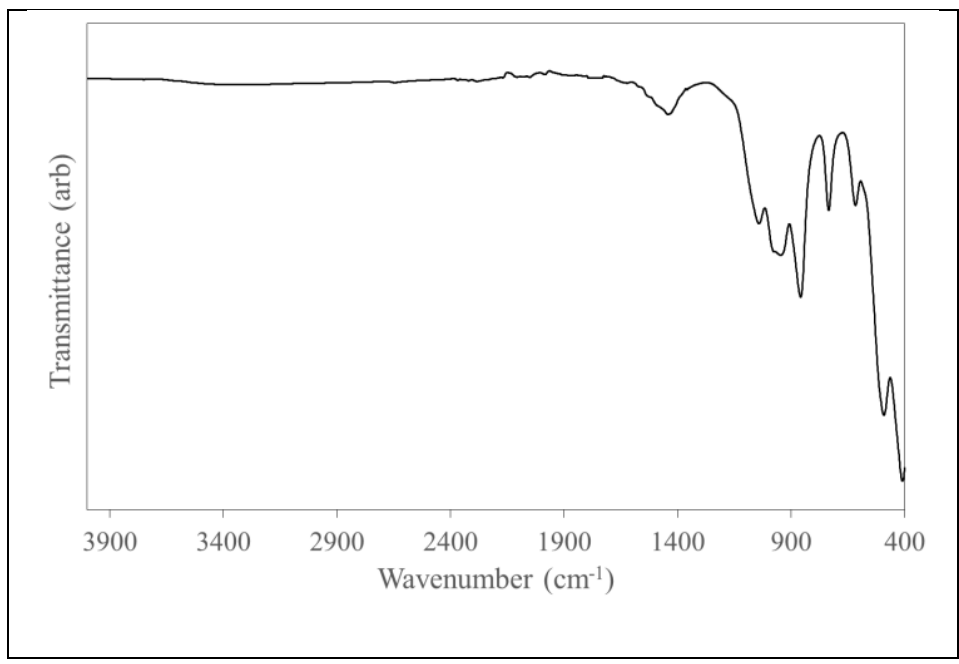

Figure 2. FTIR spectrum for the hydrothermal reaction product.

The FTIR spectrum of the lithium metasilicate reaction product is presented in Fig. 2 and closely resembles those of lithium metasilicate reported in the literature [2, 13]. Si-O-Si vibrations give rise to bands at 1045, 984, 736 and $619 \mathrm{~cm}^{-1}$. The signal at $523 \mathrm{~cm}^{-1}$ is attributed to Si-O-Li deformations and $\mathrm{O}-\mathrm{Si}-\mathrm{O}$ vibrations give rise to the bands at 945 and $863 \mathrm{~cm}^{-1}$.

\subsection{The Knoevenagel condensation reaction}

The potential ability of the lithium metasilicate product to catalyse the Knoevenagel condensation was tested on the reaction of benzaldehyde and ethyl cyanoacetate at $80{ }^{\circ} \mathrm{C}$ to produce ethyl trans- $\alpha$ cyanocinnamate. The ethyl trans- $\alpha$-cyanocinnamate product was confirmed by GC-MS, and the reaction, in the presence and absence of the catalyst, was monitored by GC. The relative concentrations of the starting reagents were used to evaluate the rate of reaction. The solution profiles of benzaldehyde and ethyl cyanoacetate as functions of time are plotted in Figure 3. These data indicate that the rate of reaction is significantly enhanced in the presence of the heterogeneous lithium metasilicate catalyst. It should be noted, that two additional by-product phases, both of relative peak intensity below $0.1 \%$, were detected by GC. These products have yet to be identified by mass spectrometry.

A previous study by Chen et al. [8], has also confirmed that lithium metasilicate derived from waste rice husk ash has been used to successfully catalyse the production of biodiesel from soybean oil with methanol. In this respect, waste-derived lithium metasilicate appears to be a promising basic heterogeneous catalyst for organic synthesis.

An investigation of the recovery and reuse potential of the SLSG-derived lithium metasilicate product for the catalysis of the Knoevenagel condensation and its ability to catalyse other organic reactions is now underway. The next stage of this research will also consider the economic feasibility of scaling-up this system.

\section{Conclusions}

This study has confirmed that a mixed product of lithium metasilicate (74\%) with minor proportions of calcium carbonate, lithium carbonate and calcium hydroxide can be prepared by hydrothermal reaction of waste green container glass in $4 \mathrm{M}$ lithium hydroxide solution at $125^{\circ} \mathrm{C}$ for 14 days. The lithium metasilicate product was found to successfully catalyse the Knoevenagel synthesis of ethyl 
trans- $\alpha$-cyanocinnamate from benzaldehyde and ethyl cyanoacetate. These findings indicate that this material is a promising basic heterogeneous catalyst for organic synthesis.

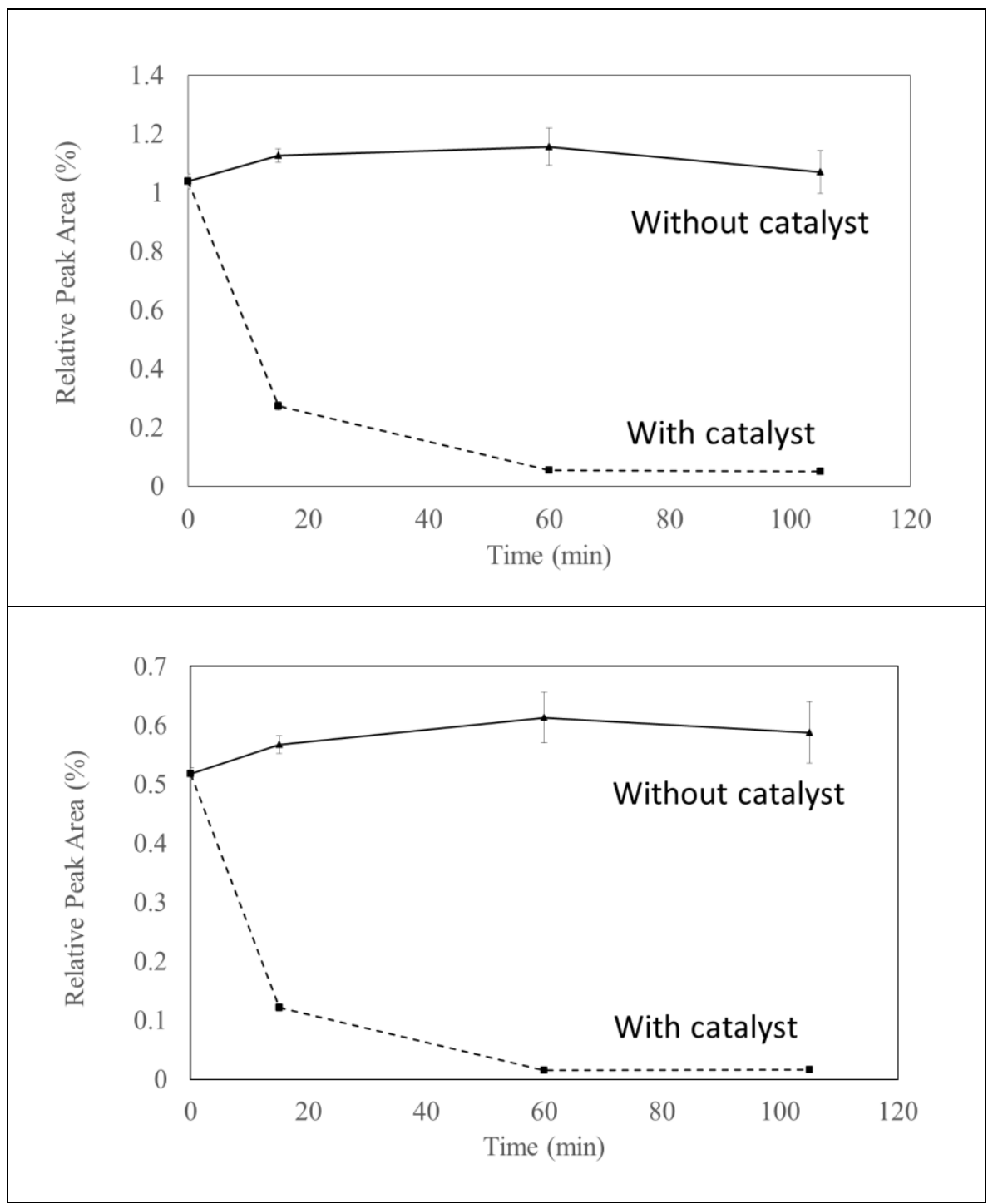

Figure 3. Concentration profiles of benzaldehyde (top) and ethyl cyanoacetate (bottom).

\section{References}

1. N.J. Coleman, Q. Li, A. Raza, Physicochem. Probl. Miner. Process. 50, 5 (2014)

2. N.J. Coleman, A.P. Hurt, A. Raza, Physicochem. Probl. Miner. Process. 51, 685 (2015)

3. F. Matteucci, M. Dondi, G. Guarini, Ceram. Int. 28, 873, (2002)

4. C.A. Velis, C. Franco-Salinas, C. O'Sullivan J. Najorka, A.R. Boccaccini, C.R. Cheeseman, Environ. Sci. Technol. 48, 7527 (2014)

5. H. Pfeifer, P. Bosch, S. Bulbulian, J. Nucl. Mater. 257, 309 (1998)

6. J. Ortiz-Landeros, M.E. Contreras-Garcia, C. Gomez-Yanez, H. Pfeifer, J. Solid State Chem. 184, 1304 (2011)

7. J.A. Bennett, K. Wilson, A.F. Lee, J. Mater. Chem. A 4, 3617 (2016) 
8. K.-T. Chen, J.-X. Wang, Y.-M. Dai, P.-H. Wang, C.-Y. Liou, C.-W. Nien, J.-S. Wu, C.-C. Chen, J. Taiwan Inst. Chem. Eng. 44, 622 (2013)

9. N.P. Tangale, S.K. Sonar, P.S. Niphadkar, P.N. Joshi, J. Ind. Eng. Chem. 40, 128 (2016)

10. R.W. Cheary, A.A. Coelho, J. Appl. Cryst. 25, 109 (1992)

11. T. Tang, P. Chen, W. Luo, D. Luo, Y. Wang, J. Nucl. Mater. 420, 31 (2012)

12. D. Jain, M. Mishra, A. Rani, Fuel Process. Technol. 95, 119 (2012)

13. B. Zhang, M. Nieuwoudt, A.J. Easteal, J. Am. Ceram. Soc. 91, 1927 (2008) 